1. The characterization of the two catalysts:

1.1 The Physical characteristics of $\mathrm{Mn}(0.4) / \mathrm{TiO}_{2}$ and $\mathrm{Fe}(0.1)-\mathrm{Mn}(0.4) / \mathrm{TiO}_{2}$

$\begin{array}{ccc}\text { Samples } & \text { BET Surface area }\left(\mathrm{m}^{2} / \mathrm{g}\right) & \text { Pore volume } \\ \left(\mathrm{cm}^{3} / \mathrm{g}\right)\end{array}$

1.2 Atomic concentrations on the surface of $\mathrm{Mn}(0.4) / \mathrm{TiO}_{2}$ and $\mathrm{Fe}(0.1)-\mathrm{Mn}(0.4) / \mathrm{TiO}_{2}$ obtained with XPS

\begin{tabular}{cccc}
\hline catalyst & \multicolumn{3}{c}{ Metal content (At\%) } \\
\cline { 2 - 4 } & $\mathrm{Fe}$ & $\mathrm{Mn}$ & $\mathrm{Ti}$ \\
\hline $\mathrm{Mn}(0.4) / \mathrm{TiO}_{2}$ & 0 & 19.4 & 20.61 \\
$\mathrm{Fe}(0.1)-\mathrm{Mn}(0.4) / \mathrm{TiO}_{2}$ & 2.43 & 28.86 & 25.61 \\
\hline
\end{tabular}

1.3 XPS measurement of the catalysts

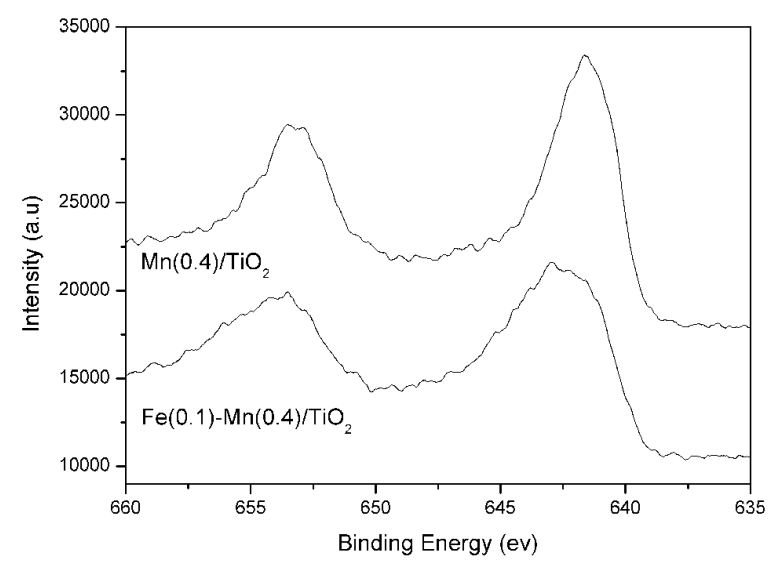

Mn 2p XPS spectra for $\mathrm{Mn}(0.4) / \mathrm{TiO}_{2}$ and $\mathrm{Fe}(0.1)-\mathrm{Mn}(0.4) / \mathrm{TiO}_{2}$ 


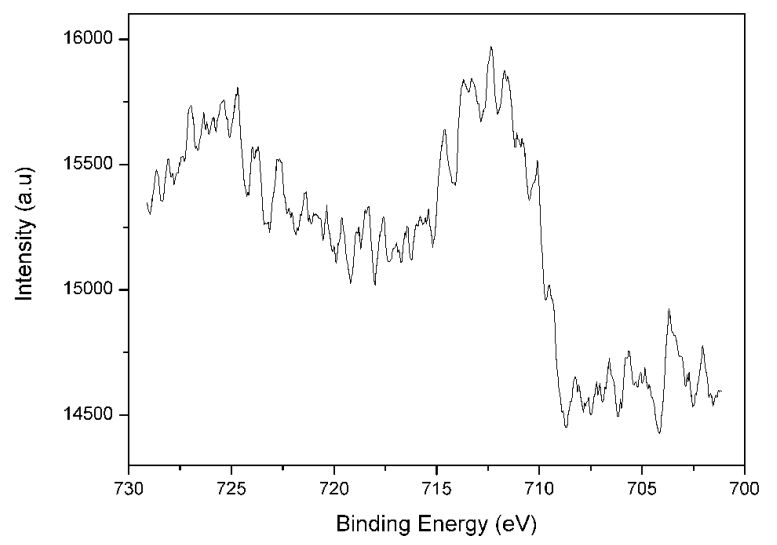

Fe 2p XPS spectra for $\mathrm{Fe}(0.1)-\mathrm{Mn}(0.4) / \mathrm{TiO}_{2}$

1.4 XRD measurement of the catalysts

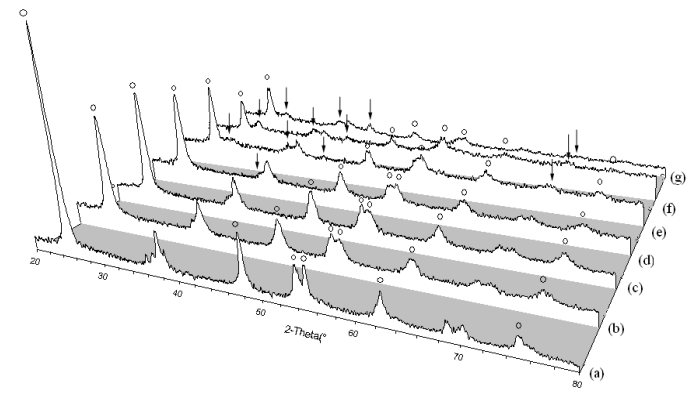

XRD spectra of (a) $\mathrm{TiO}_{2}$, (b) $\mathrm{Mn}(0.1) / \mathrm{TiO}_{2}$, (c) $\mathrm{Mn}(0.2) / \mathrm{TiO}_{2}$, (d) $\mathrm{Mn}(0.3) / \mathrm{TiO}_{2}$, (e) $\mathrm{Mn}(0.4) /$

$\mathrm{TiO}_{2}$, (f) $\mathrm{Mn}(0.5) / \mathrm{TiO}_{2},(\mathrm{~g}) \mathrm{Mn}(0.6) / \mathrm{TiO}_{2}$ (o, anatase; $\downarrow, \mathrm{MnO}_{2}$ ).

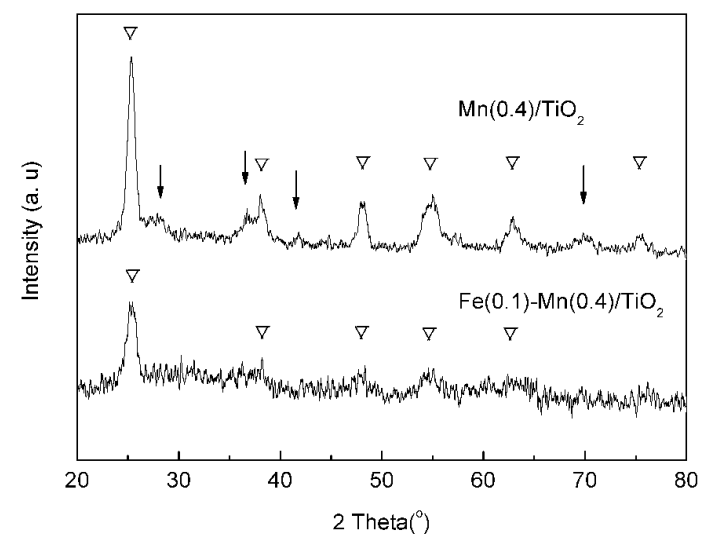

XRD spectra of $\mathrm{Mn}(0.4) / \mathrm{TiO}_{2}$ and $\mathrm{Fe}(0.1)-\mathrm{Mn}(0.4) / \mathrm{TiO}_{2}$ ( $\square$, anatase; $\downarrow, \mathrm{MnO}_{2}$ ) 
2. The frequencies of all bands and assignments.

\begin{tabular}{|c|c|c|}
\hline Samples & Frequency $(\mathrm{cm}-1)$ & assignment \\
\hline \multirow[t]{14}{*}{$\mathrm{Mn}(0.4) / \mathrm{TiO}_{2}$} & $1598,1210,1170$ & coordinated $\mathrm{NH}_{3}$ on Lewis \\
\hline & & acid sites \\
\hline & $1443,1850-1640$ & $\mathrm{NH}_{4}^{+}$species on Brønsted acid \\
\hline & & sites \\
\hline & 3349,3245 and 3149 & NH stretching \\
\hline & 966,1230 & weakly adsorbed $\mathrm{NH}_{3}$ or \\
\hline & & gas-phase $\mathrm{NH}_{3}$ \\
\hline & 2237 & $\mathrm{~N}_{2} \mathrm{O}$ \\
\hline & 1440 & nitro compounds \\
\hline & 1625 & $\mathrm{NO}_{2}$ \\
\hline & $1530,1275,1290$ & monodentate nitrate \\
\hline & 1580,1555 & bidentate nitrate \\
\hline & 1660 & $\mathrm{~N}_{2} \mathrm{O}_{4}$ \\
\hline & $1540-1570$ & amide species \\
\hline \multirow[t]{13}{*}{$\mathrm{Fe}(0.1)-\mathrm{Mn}(0.4) / \mathrm{TiO}_{2}$} & $1598,1210,1198$ & coordinated $\mathrm{NH}_{3}$ on Lewis acid \\
\hline & & sites \\
\hline & $1443,1850-1640$ & $\mathrm{NH}_{4}{ }^{+}$species on Brønsted acid \\
\hline & & sites \\
\hline & 3349,3245 and 3149 & NH stretching \\
\hline & 1170 & coordinated $\mathrm{NH}_{3}$ on $\mathrm{Fe}_{2} \mathrm{O}_{3}$ \\
\hline & 2237 & $\mathrm{~N}_{2} \mathrm{O}$ \\
\hline & 1440 & nitro compounds \\
\hline & 1625,1619 & $\mathrm{NO}_{2}$ \\
\hline & 1580,1555 & bidentate nitrate \\
\hline & 1660 & $\mathrm{~N}_{2} \mathrm{O}_{4}$ \\
\hline & 1210 & nitrites formed on $\mathrm{Fe}_{2} \mathrm{O}_{3}$ \\
\hline & $1530,1275,1290$ & monodentate nitrate \\
\hline
\end{tabular}




\section{DRIFT spectra}

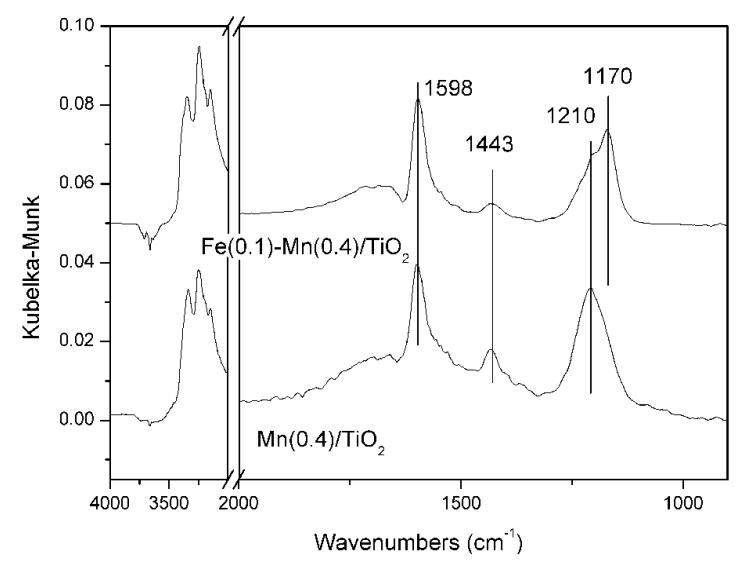

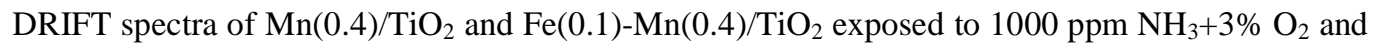
after purging by $\mathrm{He}$ for $30 \mathrm{~min}$ at $423 \mathrm{~K}$.

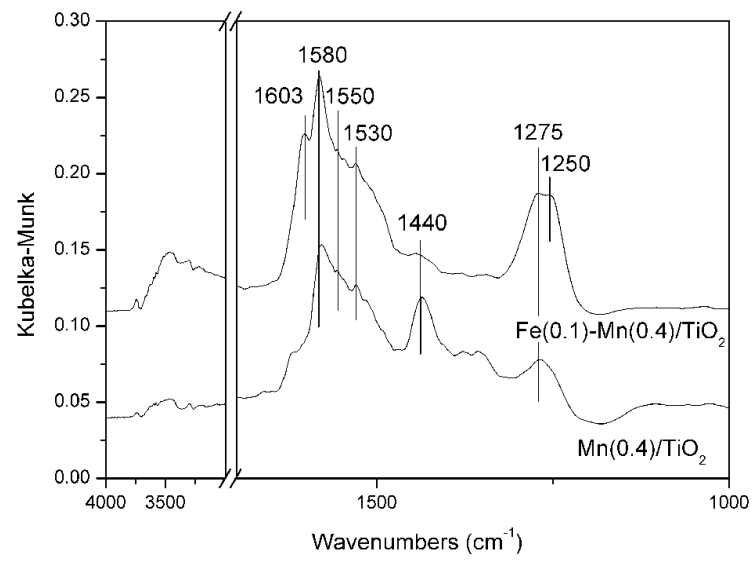

DRIFT spectra of $\mathrm{Mn}(0.4) / \mathrm{TiO}_{2}$ and $\mathrm{Fe}(0.1)-\mathrm{Mn}(0.4) / \mathrm{TiO}_{2}$ exposed to $1000 \mathrm{ppm} \mathrm{NO}+3 \% \mathrm{O}_{2}$ at $423 \mathrm{~K}$ and after purging by $\mathrm{He}$ for $30 \mathrm{~min}$. 